\title{
Perquinolines A-C: Unprecedented Bacterial Tetrahydroisoquinolines Involving an Intriguing Biosynthesis
}

\author{
Yuriy Rebets ${ }^{+}$, Suvd Nadmid ${ }^{+}$, Constanze Paulus, Charlotte Dahlem, Jennifer Herrmann, \\ Harald Hübner, Christian Rückert, Alexandra K. Kiemer, Peter Gmeiner, Jörn Kalinowski, \\ Rolf Müller, and Andriy Luzhetskyy*
}

\begin{abstract}
Metabolic profiling of Streptomyces sp. IB2014/0166 led to the identification of three new tetrahydroisoquinoline natural products, perquinolines $A-C(\mathbf{1}-3)$. Labelled precursor feeding studies and the cloning of the pqr biosynthetic gene cluster revealed that 1-3 are assembled by the action of several unusual enzymes. The biosynthesis starts with the condensation of succinyl-CoA and L-phenylalanine catalyzed by the amino7-oxononanoate synthase-like enzyme PqrA, representing rare chemistry in natural product assembly. The second condensation and cyclization events are conducted by PqrG, an enzyme resembling an acyl-CoA ligase. Last, ATP-grasp RimK-type ligase PqrI completes the biosynthesis by transferring a $\gamma$ aminobutyric acid or $\beta$-alanine moiety. The discovered pathway represents a new route for assembling the tetrahydroisoquinoline cores of natural products.
\end{abstract}

soquinoline serves as the core of various naturally occurring, synthetic and semisynthetic compounds that demonstrate a broad spectrum of biological activities. ${ }^{[1]}$ Among natural products, the plant-derived alkaloids of the benzylisoquinoline family are estimated to include approximately 800 unique chemical structures, many of which, such as morphine, papaverine, and noscapine, are of high medical importance (Figure 1).$^{[2]}$ On the other hand, bacterial tetrahydroisoquino-

[*] Dr. Y. Rebets, ${ }^{[+]}$Dr. S. Nadmid, ${ }^{[+]}$Dr. C. Paulus, Prof. Dr. A. Luzhetskyy Department of Pharmacy, Pharmaceutical Biotechnology University of Saarland

Campus, Bld. C2 3, Saarbrucken 66123 (Germany)

E-mail: a.luzhetskyy@mx.uni-saarland.de

C. Dahlem, Prof. Dr. A. K. Kiemer

Department of Pharmacy, Pharmaceutical Biology University of Saarland

Campus, Bld. C2 3, Saarbrucken 66123 (Germany)

Dr. J. Herrmann, Prof. Dr. R. Müller, Prof. Dr. A. Luzhetskyy Department Microbial Natural Products

Helmholtz Institute for Pharmaceutical Research Saarland (HIPS) Campus, Bld. 8 1, Saarbrucken 66123 (Germany)

Dr. H. Hübner, Prof. Dr. P. Gmeiner

Department of Chemistry and Pharmacy

Friedrich-Alexander-Universität Erlangen-Nürnberg

Nikolaus-Fiebiger-Strasse 1091058 Erlangen (Germany)

Dr. C. Rückert, Prof. Dr. J. Kalinowski

Center for Biotechnology-CeBiTec

University of Bielefeld

Universitätsstraße 2533615 Bielefeld (Germany)

$\left.{ }^{+}\right]$These authors contributed equally to this work.

(2) Supporting information and the ORCID identification number(s) for

(D) the author(s) of this article can be found under:

https://doi.org/10.1002/anie.201905538.
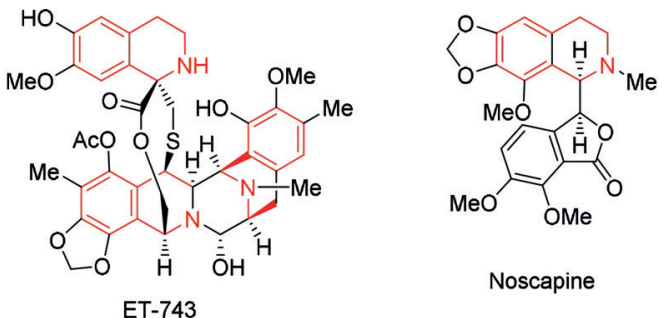

Noscapine
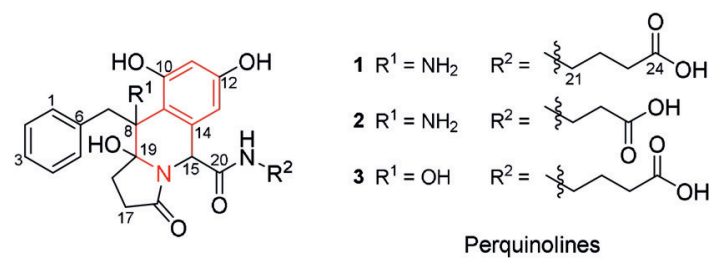

Figure 1. Structure of ecteinascidin ET-743, noscapine and perquinolines $A-C(1-3)$. Tetrahydroisoquinoline rings are highlighted in red.

line natural products are less common but still have significance as potent anticancer agents. ${ }^{[3]}$ As such, saframycins A and $\mathrm{Mx}$, quinocarcin, dnacins, and ecteinascidins have shown strong anticancer and migration inhibiting activities thought to be based on interactions with the DNA. Ecteinascidin ET743 (commercial name Trabectidin/Yondelis ${ }^{\circledR}$ ) has been approved in the EU for soft tissue carcinoma treatment and in the USA for liposarcoma (Figure 1).$^{[4]}$ Despite the differences in structures and biological sources, both plant-derived benzylisoquinoline alkaloids and bacterial tetrahydroisoquinolines originate from Pictet-Spengler reactions catalyzed by specific enzymes. ${ }^{[1 b, 5]}$ Norcoclaurine synthase catalyzes the condensation of dopamine and 4-hydroxyphenylacetaldehyde to form norcoclaurine, which is a key intermediate for many, if not all, benzylisoquinoline alkaloids. ${ }^{[6]}$ Bacterial tetrahydroisoquinolines are assembled by non-ribosomal peptide synthases. ${ }^{[7]}$ In this case, reductase with substrate promiscuity generates aldehyde substrates that are used in the PictetSpengler reaction catalyzed by the condensation domain, which is specific for amine-bearing substrates.

Herein, we describe the identification and biosynthesis of new bacterial tetrahydroisoquinolines named perquinolines A-C (1-3; Figure 1). The compounds were isolated from Streptomyces sp. IB2014/016-6 (Figure S1 in the Supporting Information) using a dereplication-based screening method aimed at the identification of new natural products. The planar structures of 1-3 were elucidated by analysis of their HR-MS and NMR spectroscopy data (Figure 1, Figure 2, Figures S2,S3 and Tables S3,S4). Perquinoline A 


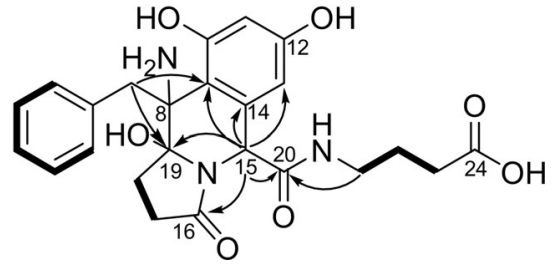

Figure 2. $2 \mathrm{D}$ NMR data of 1 showing the key HMBC (arrows) and COSY correlations (bold lines).

(1), the main product, shows an ion peak at $\mathrm{m} / \mathrm{z} 452[\mathrm{M}$ $\left.\mathrm{H}_{2} \mathrm{O}+\mathrm{H}\right]^{+}$consistent with the molecular formula $\mathrm{C}_{24} \mathrm{H}_{27} \mathrm{~N}_{3} \mathrm{O}_{7}$. Interpretation of the 1D and 2D NMR data of $\mathbf{1}$ revealed four spin systems consisting of 2-pyrrolidone and a benzyl moiety along with a dihydroxyphenyl ring and a $\gamma$ aminobutyric acid (GABA). The key HMBC correlations from $\mathrm{H}-7$ of the benzyl ring established its connectivity with the 2-pyrrolidone and dihydroxyphenyl moieties. A further HMBC correlation was observed from $\mathrm{H}-21$ of GABA to carbonyl carbon $\mathrm{C}-20$, which has a heteronuclear HMBC correlation with $\mathrm{H}-15$, leading to the extension of the GABA moiety. Isolated methine singlet $\mathrm{H}-15$ showed key HMBC correlations to the 2-pyrrolidone and dihydroxyphenyl moieties, forming the tetrahydroisoquinoline ring and completed the planar structure of $\mathbf{1}$ (Figure 2).

Perquinoline B (2) showed an ion at $m / z 438\left[M-\mathrm{H}_{2} \mathrm{O}+\right.$ $\mathrm{H}]^{+}$, which is similar to that of $\mathbf{1}$ but suggests a shorter side chain, namely, a $\beta$-alanine moiety. Finally, perquinoline $\mathrm{C}(\mathbf{3})$ is a minor product and differs from $\mathbf{1}$ by the presence of a hydroxy group instead of an amino group at C-8 $(\mathrm{m} / \mathrm{z} 453$ $\left[M-\mathrm{H}_{2} \mathrm{O}+\mathrm{H}\right]^{+}$) (Figure 1, Figures $\mathrm{S} 1, \mathrm{~S} 3$ ). All three congeners have a core structure consisting of a 1,2,3,4-tetrahydroisoquinoline ring fused with 2-pyrrolidone and benzyl moieties and a $\gamma$-aminobutyric acid or a $\beta$-alanine as a side chain (Figure 1).Compounds 1-2 have no antibacterial activity in the concentration range tested (up to $64 \mu \mathrm{g} \mathrm{mL}^{-1}$, Table S5). These compounds demonstrated weak anticancer activity against lung and liver adenocarcinoma cell lines (Table S6). Since 1-3 share the same core structure as opiates, we tested the binding of $\mathbf{1}$ to human $\beta_{2}$-adrenergic and $\mu$-opioid receptors. ${ }^{[8]}$ Indeed, weak binding with $K_{\mathrm{i}}$ values in the tens of $\mu \mathrm{M}$ range was detected with $\mathbf{1}$ (Table S7).

The structures of 1-3 could be considered cyclized tripeptide with Phe as the N-terminal amino acid and GABA or $\beta$-alanine as the C-terminal moiety (Figure 1 ). However, the origin of the ring system of $\mathbf{1}-\mathbf{3}$ was not clear. To elucidate the biosynthetic pathway of 1-3, a culture of Streptomyces sp. IB2014/016-6 was supplemented with L[ring- $\left.d_{5}\right]$-Phe, L- $\left[2,3,3,4,4-d_{5}\right]-$ Glu, L- $\left[{ }^{13} \mathrm{C}_{4},{ }^{15} \mathrm{~N}_{2}\right]$-Asn and $\left[2,2,3,3-d_{4}\right]$ succinate. LC-MS data of the extracts prove that the benzyl ring of $\mathbf{1 -} \mathbf{3}$ is derived from phenylalanine (Figure S4). In the case of $\mathrm{L}-\left[{ }^{13} \mathrm{C}_{4},{ }^{15} \mathrm{~N}_{2}\right]$-Asn, the isotopic pattern suggested that most likely only the nitrogen is incorporated into 1-3, indicating the involvement of an aminotransferase at some step during biosynthesis (Figure S4). L-[2,3,3,4,4- $\left.d_{5}\right]$-Glu and $\left[2,2,3,3-d_{4}\right]$-succinate were not incorporated; thus, the origin of the 2-pyrrolidone ring and the GABA fragment of $\mathbf{1}$ and $\mathbf{3}$ remained unclear at this stage.
The remaining structural features of $\mathbf{1}-\mathbf{3}$ suggest a polyketide origin. Streptomyces sp. IB2014/016-6 was thus grown in the presence of $\left[1-{ }^{13} \mathrm{C}\right]-,\left[2-{ }^{13} \mathrm{C}\right]$-, and $\left[1,2-{ }^{13} \mathrm{C}_{2}\right]$-sodium acetates. Compound 1 was purified, and ${ }^{13} \mathrm{C}$ NMR spectroscopy was used to determine the positions of acetate incorporation (Figure 3, Table S7). The $\left[1,2-{ }^{13} \mathrm{C}_{2}\right]$-acetate incorpora-

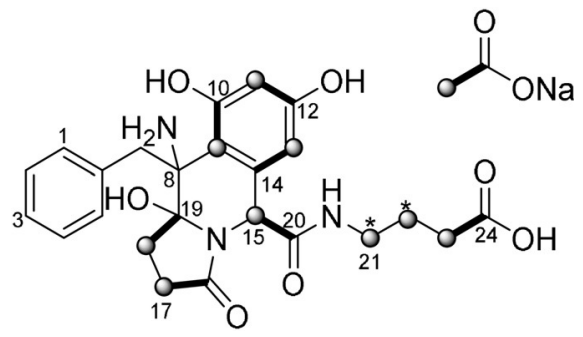

Figure 3. Incorporation of carbon-labelled sodium acetate into 1. Bold lines indicate the intact acetate units; sphere indicate the C-2 of acetate, * indicates a low rate of incorporation.

tion study clearly demonstrated that the dihydroxyphenyl ring of the tetrahydroisoquinoline originates from acetate units via a polyketide pathway. At the same time, a low level of incorporation of acetate $-{ }^{13} \mathrm{C}_{2}$ was observed at C-18 and C-19 of the 2-pyrrolidone ring. The ${ }^{13} \mathrm{C}$ NMR spectrum of purified 1 obtained from the cultivation of Streptomyces sp. IB2014/ 016-6 with $\left[1-{ }^{13} \mathrm{C}\right]$-acetate exhibited enhanced signals corresponding to carbons C-10, 12, 14, 16, 19, 20, and 24 (Table S8). Furthermore, the feeding of $\left[2-{ }^{13} \mathrm{C}\right]$-acetate resulted in enhanced signals at positions C-9, 11, 13, 15, 17, and 18. Interestingly, these data suggest a tail-to-tail connection (C2$\mathrm{C} 2$ ) of acetates to form the 2-pyrrolidone ring (positions $\mathrm{C}-17$ and $\mathrm{C}-18$ ). Such an arrangement might arise from the incorporation of succinate built up in the citric acid or the glyoxylate cycles. ${ }^{[9]}$ Moreover, C-21 and C-22 were also enriched but at a relatively lower rate, indicating the indirect incorporation of acetate into GABA (Figure 3). This suggests the GABA fragment originates from the succinate (citric acid cycle) or acetoacetate (butanoate metabolism) rather than decarboxylation of L-Glu.

To identify the 1-3 biosynthetic gene cluster, the genome of Streptomyces sp. IB2014/016-6 was sequenced and analyzed with antiSMASH. ${ }^{[10]}$ The gene cluster encoding putative ATPgrasp RimK-type ligase $(p r q I),{ }^{[1]}$ hydratase $(p q r H)$, acylCoA ligase $(p q r G)$, 8-amino-7-oxononanoate synthase (AONS, pqrA) as well as type III PKS ( $p q r B)$, two dehydratases (pqrC,D), and aminotransferase (pqrE), which are similar to Dpg enzymes involved in 3,5-dihydroxyphenylglycine (3,5-DHPG) assembly in glycopeptide biosynthesis, ${ }^{[12]}$ were proposed to be responsible for the assembly of 1-3 (Figure 4, Table S9). In addition, genes encoding putative transcriptional regulator (pqrR), transporter $(p q r W)$ and large protein with TolB and amidase conserved domain motifs (pqrF) are located in the same region of the chromosome. A BLAST search with the pqrA sequence revealed a similar gene cluster within the genome of S. odonnellii NRRL B24891 (Figure S5). This strain, when cultivated under the same conditions used herein for Strepto- 


\begin{tabular}{|l|l|}
\hline Gene & Putative product \\
\hline$p q r F$ & $\begin{array}{l}\text { Translocation protein TolB with amidase } \\
\text { domain }\end{array}$ \\
\hline$p q r E$ & Transaminase \\
\hline$p q r D$ & Hydratase \\
\hline$p q r C$ & Hydratase \\
\hline$p q r B$ & Alpha-pyrone synthesis \\
\hline$p q r A$ & $\begin{array}{l}\text { 2-amino-3-ketobutyrate coenzyme A } \\
\text { ligase; 8-amino-7-oxononanoate synthase }\end{array}$ \\
\hline$p q r G$ & Phenylacetate-coenzyme A ligase \\
\hline$p q r H$ & Hydratase \\
\hline$p q r R$ & Regulatory proteins, LuxR family \\
\hline$p q r W$ & Major Facilitator Superfamily protein \\
\hline$p q r l$ & Glutathione synthetase, RimK \\
\hline
\end{tabular}

Figure 4. Organization pqr gene cluster and predicted functions of prq genes.

myces sp. IB2014/016-6, was found to produce 1-3 (Figure S6). To confirm that the proposed gene cluster is involved in the biosynthesis of 1-3, the pqrA gene was deleted from the chromosome of IB2014/016-6. The resulting mutant did not produce 1-3 (Figure S7). Lastly, we retrieved both $p q r$ gene clusters from the chromosomes of IB2014/016-6 and NRRL B24891 using the TAR cloning technique and successfully expressed them heterologously in S. lividans TK24, and the recombinant strains produced $\mathbf{1}-\mathbf{3}$ (Figure S8). ${ }^{[13]}$

Based on the precursor feeding experiments, we proposed that the entire biosynthesis of $\mathbf{1}-\mathbf{3}$ starts with the condensation of Phe with succinate rather than succinate being introduced into the assembled peptide later. The obvious candidate protein for this biosynthetic step is the putative 8-amino-7oxononanoate synthase (AONS) homologue encoded by the pqrA gene. This group of enzymes catalyzes the decarboxylative condensation of amino acid and acyl-CoA substrates in the first steps of the biotin and haem biosynthesis. ${ }^{[13]}$ Recently, an unusual polyketide synthase employing AONStype reactions was reported to assemble the core of saxitoxin. ${ }^{[14]}$ Additionally, AONS was proposed to participate in the production of ring $\mathrm{A}$ of moenomycin and other bacterial natural products. ${ }^{[15]}$ Pyridoxal phosphate (PLP) is a crucial cofactor for these enzymes. We expressed pqrA in E. coli and purified the corresponding protein (Figure S9). The PqrA $(1.5 \mu \mathrm{M})$ activity was tested with $5 \mathrm{~mm}$ L-Phe, $1.2 \mathrm{~mm}$ succinyl$\mathrm{CoA}$, and $0.1 \mathrm{~mm}$ PLP at $37^{\circ} \mathrm{C}$. The reaction mixture with boiled enzyme was used as a control (Figure S10a). The mixture was analyzed by LC-MS (Figure 5a). As a result, a peak with $m / z, 222[M+\mathrm{H}]^{+}$that is not present in the control appeared in the spectrum of the reaction mixture containing active PqrA (compound 4). The molecular weight of compound $\mathbf{4}$ corresponds to the structure of the proposed product of the PrqA reaction (detected $m / z 222.1124[M+\mathrm{H}]^{+}$, calculated $\mathrm{m} / z 222.112376[\mathrm{M}+\mathrm{H}]^{+}$; Figure $5 \mathrm{~b}$ ). Compound 4 is formed by the decarboxylative condensation of L-Phe and succinyl-CoA. One additional compound with $\mathrm{m} / z 266$ $[M+\mathrm{H}]^{+}(\mathbf{5})$ is present in the reaction mixtures with the active and denatured PqrA (Figure 5c). Compound 5 was purified, and its structure was determined by NMR spectros- a

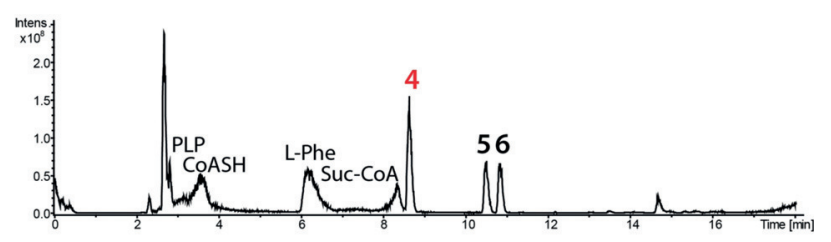

b

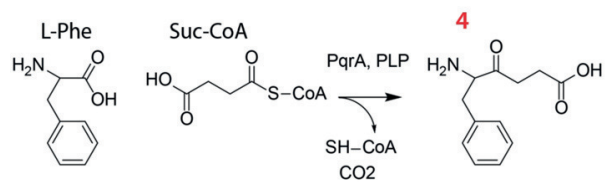

$c$
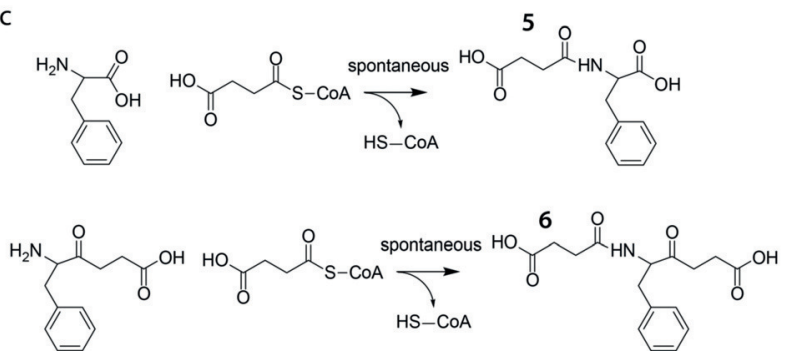

Figure 5. PqrA catalyzed the reaction and spontaneous acylation of LPhe and 4. a) BPC chromatogram of the PqrA reaction mixture. b) Proposed reaction catalyzed by PqrA. c) Proposed acylation reactions of $\mathrm{L}-\mathrm{Phe}$ and 4 . Suc-CoA $=$ succinyl-CoA; PLP $=$ pyridoxal phosphate.

copy (Table S10). This compound is an adduct formed by the spontaneous acylation of the $\alpha$-amino group of L-Phe with succinate. When tested, $\mathbf{5}$ is not accepted as a substrate by PqrA. Furthermore, in the reaction mixture with active PqrA, a compound with $m / z 322[M+\mathrm{H}]^{+}(\mathbf{6})$ can be detected. The mass of $\mathbf{6}$ corresponds to an acylation adduct similar to $\mathbf{4}$ (Figure S10b).

We have analyzed the properties of PqrA catalysis. The maximum rate of substrate conversion was achieved at $37^{\circ} \mathrm{C}$ after 2 hours of incubation (Figure S11). The rate of the enzymatic reaction was found to be strongly dependent on the concentration of PLP in the reaction mixture (Figure S12). When no PLP was added, only conversion rates of 3-8\% relative to the maximum activity were observed. The residual activity can be explained by the fact that some of the protein was already covalently modified with PLP during production in E. coli. To confirm the mechanism of the reaction with PqrA, $1-{ }^{13} \mathrm{C}$ and $2-{ }^{13} \mathrm{C}$ L-Phe were used. With $2-{ }^{13} \mathrm{C} \mathrm{L}-\mathrm{Phe}$, the major peak in the mass spectrum of $\mathbf{4}$ was shifted to $\mathrm{m} / \mathrm{z} 223$ $[M+\mathrm{H}]^{+}$(Figure S13). However, when $1-{ }^{13} \mathrm{C}$ L-Phe was used, no increase in the $\mathrm{m} / \mathrm{z}$ of $\mathbf{4}$ was observed, leading to the conclusion that the carboxyl group of L-Phe is removed during the condensation reaction, as described for other AONS. $^{[16]}$

We also tested the substrate specificity of PqrA for both amino acid and acyl-CoA substrates. The enzyme accepted glutaryl-CoA but with much lower efficiency than succinylCoA (Figure S14a). At the same time, PqrA does not accept butyryl-CoA or methylmalonyl-CoA with the same number of carbon atoms as succinyl-CoA, nor does it accept malonyl- 
CoA or acetyl-CoA. PqrA is highly specific for L-amino acids and cannot act on D-Phe (Figure S14b). Of the 15 tested proteinogenic amino acids, PqrA was able to catalyze the condensations of L-Phe, L-Tyr, and L-Met (Figure S14b). Consequently, we were able to detect the L-Tyr perquinoline derivative (7) in the extracts of the native producer and in recombinant S. lividans strains carrying the prq gene cluster (Figures S15 and S16). PqrA was not able to accept L-phenyllactate as a substrate, which suggests that $\mathbf{3}$ is a shunt product in the biosynthesis of $\mathbf{1}$ and 2. Owing to the amino-acid substrate promiscuity of PqrA, we hypothesized that it might accept other derivatives of L-Phe. Indeed, PqrA was able to catalyze the reactions of L-Phe derivatives bearing substituents on the benzyl ring but not at the $\alpha$-carbon or $\alpha$-aminogroup (Figure S17).

Last, a model for the PqrA structure was generated with SWISS-Model using E. coli 2-amino-3-ketobutyrate coenzyme A ligase (1fc4.1.A) as the template (Figure S18). ${ }^{[1]}$ Based on this model, we predicted and mutated the amino acids contributing to the formation of the PqrA active site, including the highly conserved Lys 265, which is assumed to be responsible for PLP binding (Table S11). To our great surprise, the Lys265His variant retained residual activity (up to $0.5 \%$ from the wild type PqrA), whereas other mutations of this amino acid showed no reactivity.

To elucidate subsequent steps in the assembly of $\mathbf{1 - 3}$, we deleted genes within and surrounding the $p q r$ gene cluster and analyzed the metabolites produced by recombinant $S$. lividans strains carrying the mutated constructs. Deletion of gene 28335 , encoding putative epoxidase on the left shoulder of the pqr cluster, and gene 28395, encoding rRNA methyltransferase on the right shoulder, have no influence on the production of 1-3 (Table 1, Figure S19). The same effect was observed with the deletion of the $p q r F$ gene. As expected, inactivation of $\operatorname{prq} A, B$, and $E$ caused complete cessation of 1-3 biosynthesis since the corresponding enzymes are responsible for the initial condensation and assembly of 3,5-DHPG (Table 1, Figure S20). Importantly, the deletion of pqrI, encoding ATP-grasp protein, caused a lack of 1-3 but did result in the accumulation of intermediate $\mathbf{8}$, which constitutes the core of $\mathbf{1}$ and $\mathbf{2}$ without the C-terminal amino acids (Table 1, Figures S21,S22). This result shows that PqrI, like similar enzymes, decorates $\mathbf{1}-\mathbf{3}$ by transferring $\gamma$-aminobutyric or $\beta$-aminopropionic acid moieties. ${ }^{[18]}$ Surprisingly, deletion of the pqrR-encoding transcription regulator also led to the production of $\mathbf{8}$, indicating the possibility that PqrR positively regulates the final modification and potentially the transport of $\mathbf{1}-\mathbf{3}$.

Last, recombinant $S$. lividans carrying the $p q r$ cluster lacking the $p q r G$ gene was found to accumulate $\mathbf{4}$ but not 1-3 (Figure S23). PqrG is similar to acyl-CoA ligases and shows some degree of homology to the A-domains of NRPS enzymes, including those implicated in the PictetSpengler reactions in tetrahydroisoquinoline biosynthesis (Figure S24) ${ }^{[19]}$ This sim-
Table 1: Accumulations of intermediates in the biosynthesis of 1-3 by mutants lacking individual pqr genes.

\begin{tabular}{llll}
\hline $\begin{array}{l}\text { Gene } \\
\text { deleted }\end{array}$ & $\begin{array}{l}\text { Compound pro- } \\
\text { duced }\end{array}$ & $\begin{array}{l}\text { Gene } \\
\text { deleted }\end{array}$ & $\begin{array}{l}\text { Compound pro- } \\
\text { duced }\end{array}$ \\
\hline 28335 & $\mathbf{1 - 3}$ & prqC & $\mathbf{4}$ \\
prqA & none & prqH & $\mathbf{1 - \mathbf { 3 } ^ { [ \mathrm { a } ] }}$ \\
prqB & none & prqR & $\mathbf{8}$ \\
prqE & none & prql & $\mathbf{8}$ \\
prqF & $\mathbf{1 - 3}$ & 28395 & $\mathbf{1 - 3}$ \\
\hline
\end{tabular}

[a] 4-6-fold increase in production.

ilarity leads to the assumption that PqrG catalyzes the condensation reaction between $\mathbf{4}$ and 3,5-DHPG. The final cyclizations of 1-3 remain unclear since the deletion of $\mathrm{pqrH}$, the last possible candidate for this reaction, led to a significant increase in the production of $\mathbf{1 - 3}$ (Figure S25). However, we believe that the observed phenotype is caused by the insertion of the resistance cassette with the strong promoter, driving the transcription of downstream genes. To identify the enzyme responsible for the last cyclization, we cloned pqrG in combination with pqrA (to provide 4) both with and without pqrH. The corresponding constructs were expressed from the ErmE promoter in S. lividans. After feeding 3,5-DHPG, the strain carrying pqrG and pqrA was able to accumulate $\mathbf{8}$ regardless of the presence of $p q r H$ (Figure S26). This result suggests that PqrG is responsible for the condensation of $\mathbf{4}$ and 3,5-DHPG and the formation of both rings of $\mathbf{1 - 3}$. The formation of $\mathbf{3}$ involves rearrangements in close proximity to the amino group of L-Phe, which seems to result in its displacement with water, leading to the formation of shunt product 3 .

Based on the obtained data, we can deduce the biosynthetic steps leading to the production of $\mathbf{1 - 3}$ (Scheme 1). First, PqrA catalyzes the condensation between L-Phe and succinylCoA, leading to intermediate 4. PqrB-E produce 3,5-DHPG, which is condensed with 4 by PqrG. The cyclization between the nitrogen atom of the peptide bond and C-19 of the carbonyl group most likely occurs spontaneously, followed by PqrG catalyzed closure of the piperidine ring, giving intermediate $\mathbf{8}$. This compound is further modified by the addition of GABA or $\beta$-alanine by PqrI, leading to 1-2. The loss of the

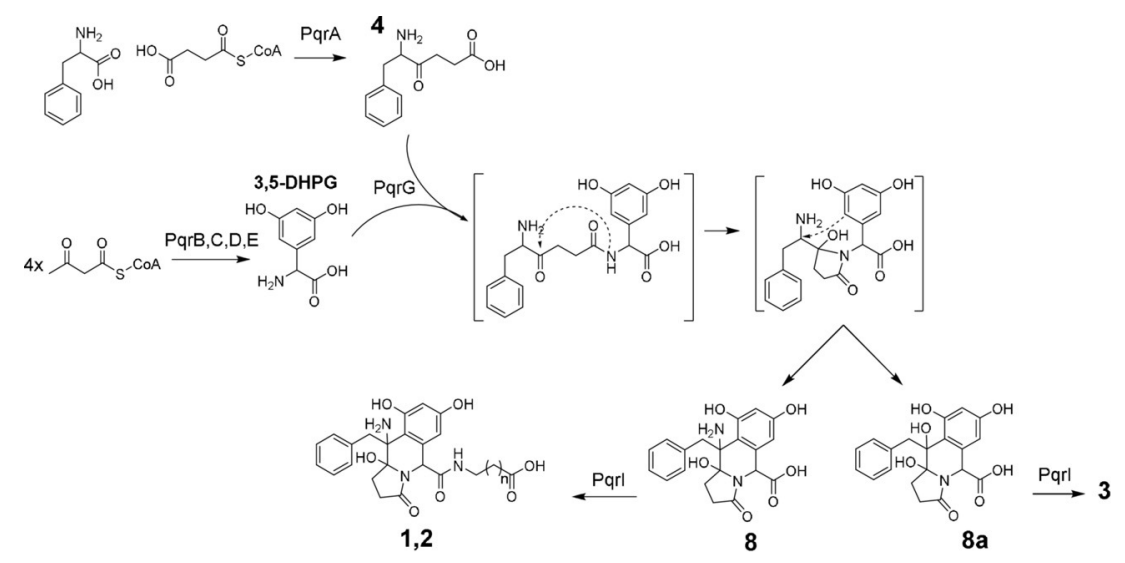

Scheme 1. Proposed biosynthetic pathway for 1-3. 
amino group during the cyclization results in the production of 3 .

In conclusion, 1-3 are a new type of tetrahydroisoquinoline natural product. Although 1-3 did not show any prominent activity in the assays employed, the biosynthetic pathway leading to the formation of these compounds represents an unprecedented assembly of the tetrahydroisoquinoline core structure. Furthermore, the biosynthesis of 1-3 broadens our understanding of the involvement of AONS and ATP-grasp proteins in the assembly of natural products.

\section{Acknowledgements}

We thank ARS Culture Collection (NRRL) for providing $S$. odonnellii NRRL B24891. We are also grateful to Prof. Uli Kazmaier for discussion of the biosynthetic steps. We would like to express many thanks to Dr. Silke Wenzel for the help with deciphering biosynthesis and preparing manuscript.

\section{Conflict of interest}

The authors declare no conflict of interest.

Keywords: 8-amino-7-oxononanoate synthase - biosynthesis . natural product . Streptomyces - tetrahydroisoquinoline

How to cite: Angew. Chem. Int. Ed. 2019, 58, 12930-12934 Angew. Chem. 2019, 131, 13063-13068

[1] a) P. L. Schiff, J. Nat. Prod. 1991, 54, 645-749; b) J. Stöckigt, A. P. Antonchick, F. R. Wu, H. Waldmann, Angew. Chem. Int. Ed. 2011, 50, 8538-8564; Angew. Chem. 2011, 123, 8692-8719; c) C. Weber, T. Opatz, Alkaloids Chem. Biol. Perspect. 2019, 81, $1-114$.

[2] D. Singla, A. Sharma, J. Kaur, B. Panwar, G. P. Raghava, $B M C$ Pharmacol. 2010, 10, 4.

[3] J. D. Scott, R. M. Williams, Chem. Rev. 2002, 102, 1669-1730.

[4] V. H. Le, M. Inai, R. M. Williams, T. Kan, Nat. Prod. Rep. 2015 $32,328-347$.

[5] G. L. Tang, M. C. Tang, L. Q. Song, Y. Zhang, Curr. Top. Med. Chem. 2016, 16, 1717-1726.

[6] a) J. M. Finefield, D. H. Sherman, M. Kreitman, R. M. Williams, Angew. Chem. Int. Ed. 2012, 51, 4802-4836; Angew. Chem. 2012, 124, 4886-4920; b) A. Bonamore, M. Barba, B. Botta, A. Boffi, A. Macone, Molecules 2010, 15, 2070-2078.

[7] K. Koketsu, K. Watanabe, H. Suda, H. Oguri, H. Oikawa, Nat. Chem. Biol. 2010, 6, 408-410.

[8] a) A. Manglik, H. Lin, D. K. Aryal, J. D. McCorvy, D. Dengler, G. Corder, A. Levit, R. C. Kling, V. Bernat, H. Hubner, X. P. Huang, M. F. Sassano, P. M. Giguere, S. Lober, D. Duan, G.
Scherrer, B. K. Kobilka, P. Gmeiner, B. L. Roth, B. K. Shoichet, Nature 2016, 537, 185; b) D. M. Rosenbaum, C. Zhang, J. A. Lyons, R. Holl, D. Aragao, D. H. Arlow, S. G. F. Rasmussen, H. J. Choi, B. T. DeVree, R. K. Sunahara, P. S. Chae, S. H. Gellman, R. O. Dror, D. E. Shaw, W. I. Weis, M. Caffrey, P. Gmeiner, B. K. Kobilka, Nature 2011, 469, 236-240.

[9] M. Hashimoto, H. Komatsu, I. Kozone, H. Kawaide, H. Abe, M. Natsume, Biosci. Biotechnol. Biochem. 2005, 69, 315-320.

[10] T. Weber, K. Blin, S. Duddela, D. Krug, H. U. Kim, R. Bruccoleri, S. Y. Lee, M. A. Fischbach, R. Muller, W. Wohlleben, R. Breitling, E. Takano, M. H. Medema, Nucleic Acids Res. 2015, 43, W237-W243.

[11] L. M. Iyer, S. Abhiman, A. M. Burroughs, L. Aravind, Mol. Biosyst. 2009, 5, 1636-1660.

[12] a) V. Pfeifer, G. J. Nicholson, J. Ries, J. Recktenwald, A. B. Schefer, R. M. Shawky, J. Schroder, W. Wohlleben, S. Pelzer, J. Biol. Chem. 2001, 276, 38370-38377; b) H. Chen, C. C. Tseng, B. K. Hubbard, C. T. Walsh, Proc. Natl. Acad. Sci. USA 2001, 98 , $14901-14906$

[13] a) P. M. Shoolingin-Jordan, S. Al-Daihan, D. Alexeev, R. L. Baxter, S. S. Bottomley, I. D. Kahari, I. Roy, M. Sarwar, L. Sawyer, S. F. Wang, Biochim. Biophys. Acta Proteins Proteomics 2003, 1647, 361-366; b) S. Mann, O. Ploux, Biochim. Biophys. Acta Proteins Proteomics 2011, 1814, 1459-1466; c) O. Bilyk, O. Sekurova, S. Zotchev, A. Luzhetskyy, Plos One 2016, 11, e0158682.

[14] S. W. Chun, M. E. Hinze, M. A. Skiba, A. R. H. Narayan, J. Am. Chem. Soc. 2018, 140, 2430-2433.

[15] a) B. Ostash, A. Saghatelian, S. Walker, Chem. Biol. 2007, 14, 257-267; b) K. Petrickova, A. Chronakova, T. Zelenka, T. Chrudimsky, S. Pospisil, M. Petricek, V. Kristufek, Front. Microbiol. 2015, 6, 814.

[16] a) I. Astner, J. O. Schulze, J. van den Heuvel, D. Jahn, W. D. Schubert, D. W. Heinz, EMBO J. 2005, 24, 3166-3177; b) D. Alexeev, M. Alexeeva, R. L. Baxter, D. J. Campopiano, S. P. Webster, L. Sawyer, J. Mol. Biol. 1998, 284, 401-419.

[17] a) A. Waterhouse, M. Bertoni, S. Bienert, G. Studer, G. Tauriello, R. Gumienny, F. T. Heer, T. A. P. de Beer, C. Rempfer, L. Bordoli, R. Lepore, T. Schwede, Nucleic Acids Res. 2018, 46, W296-W303; b) A. Schmidt, J. Sivaraman, Y. Li, R. Larocque, J. A. Barbosa, C. Smith, A. Matte, J. D. Schrag, M. Cygler, Biochemistry 2001, 40, 5151-5160.

[18] a) N. M. Llewellyn, Y. Li, J. B. Spencer, Chem. Biol. 2007, 14, 379-386; b) G. Zhao, Z. Jin, Y. Wang, N. M. Allewell, M. Tuchman, D. Shi, Proteins Struct. Funct. Bioinf. 2013, 81, $1847-$ 1854 ; c) K. Tabata, H. Ikeda, S. Hashimoto, J. Bacteriol. 2005, 187, 5195-5202; d) J. A. Baccile, J. E. Spraker, H. H. Le, E. Brandenburger, C. Gomez, J. W. Bok, J. Macheleidt, A. A. Brakhage, D. Hoffmeister, N. P. Keller, F. C. Schroeder, Nat. Chem. Biol. 2016, 12, 419-424.

[19] A. Pospiech, J. Bietenhader, T. Schupp, Microbiology 1996, 142, $741-746$

Manuscript received: May 4, 2019

Accepted manuscript online: July 16, 2019

Version of record online: August 21, 2019 